\title{
Teaching Operating Systems Scheduling
}

\author{
Shimon Cohen \\ MLA Academic Learning Center, Israel \\ shamon51@gmail.com
}

\begin{abstract}
The Operating System is a very complex program that runs on our computer (probably the most complex ...). It is difficult to comprehend the diversity of its operations, let alone - teach it. Second/Third year students with beginner programming skills are overwhelmed by the OS size and its multiple tasks.

This paper is focused on how-to better teach the operation of the OS Scheduler that manages the user's processes. The scheduler tasks are: create the process, load it into memory, allocate CPU time-slices for its execution, handle keyboard clicks and menu selection, swap the process in and out of memory, and instructs the devices to satisfy IO requests.

The question is: "how can we give the students hands-on experience on the scheduler operation? "Obviously, a few slides and a state-machine diagram, is not enough.

The answer consists of: explanation (slides), simulation and an exercise.

The simulation package includes two simulators (two levels of complexity) each running userdesigned scenarios. The $1^{\text {st }}$ simulator (A) is a simple Round-Robin scheduler that is easy to follow. The $2^{\text {nd }}$ simulator (B) comes much closer to a real OS scheduler (with all its complexity); as such it is very difficult for the student to understand, unless they play with Simulator-A first.

The exercise goal is to write a "scheduler" that allocates the "CPU" time among ten "Processes" each doing a "long" task. The exercise can be accomplished by $2^{\text {nd }}-3^{\text {rd }}$ year students.
\end{abstract}

Keywords: Operating System, Simulator, Teaching

\section{Introduction}

\section{Operating System}

The computer is inherently a parallel machine; it consists of several CPUs, Memory units, IO Controllers. Each component is in fact a basic-computer (my definition) with a CPU, logic (program), some memory and IO connectors. For example: a Disk-Controller is made of a CPU

Material published as part of this publication, either on-line or in print, is copyrighted by the Informing Science Institute. Permission to make digital or paper copy of part or all of these works for personal or classroom use is granted without fee provided that the copies are not made or distributed for profit or commercial advantage AND that copies 1) bear this notice in full and 2) give the full citation on the first page. It is permissible to abstract these works so long as credit is given. To copy in all other cases or to republish or to post on a server or to redistribute to lists requires specific permission and payment of a fee. Contact 0HPublisher@InformingScience.org to request redistribution permission. (called Controller), programmable logic usually stored in EEPROM, memory for data buffers and IO connectors that enables it to control the spinning disk, move the read/write head and communicate with other devices or the mother board. Today these components are fabricated on one chip (the so called "computer on a chip")

On top of the computer parallel/realtime hardware architecture, there are 
multiple software processes that compete on the use of the computer resources (components). The goal of the Operating System (OS) scheduler is to divide/allocate/arbitrate the use of these resources in the most efficient possible way.

The CPU is the only component that can execute programs; as such it must "wear two hats": (1) executing the multiple users programs, (2) managing the computer using the OS programs (Kernel). To do that, the CPU is constantly doing "context switch" among all its "obligations".

IO controllers are also required to do multiple tasks. For example: a Disk controller is instructed to look for several blocks, on different tracks, for several processes. Or, the network controller must send/receive different packets for multiple browser windows.

\section{Problem / Question / Answer}

The students have a problem grasping the full complexity of the computer operations. Being $2^{\text {nd }} / 3^{\text {rd }}$ year students, they write simple programs $(\mathrm{C}, \mathrm{C}++)$ that run, presumably alone, on the computer without parallelism or threads. The students are not fully aware of the computer realtime nature, and the BIG roll and many services provided by the operating system. They struggle with the idea that the CPU is very fast but also very "dumb", and that it needs very detailed instructions on how and what to execute.

As for the lecturer: presenting slides, writing on the board and being a better lecturer are the first necessary step, BUT usually the students quickly "lose contact" with the material. There are too many new ideas / concepts and they fail to "see" through it.

The question is how we can let them "see inside" the Scheduler and "feel" the operation of the OS, CPU, and IO Controllers.

The answer is: lecture (in two steps), a pair of simulators (in increasing levels of complexity) and an exercise in writing a small "scheduler". I suggest the following time line:

1. Introduction to OS, Interrupts, Processes States

2. Show the $1^{\text {st }}$ simulator

3. Explain System Calls, Timing and Scheduling

4. Show the $2^{\text {nd }}$ simulator

5. Present the Exercise

\section{Introduction to the OS and the Scheduler}

This paper is not about Operating Systems; it is about how to teach OS (in particular the Scheduler). In order to run (and understand ...) the two simulators, and do the exercise, the lecturer must cover the following topics:

- Computer architecture: CPU, Clock, BUS, Memory, IO controllers

- CPU: very ${ }^{*}$ quick (speed of light) $\leftrightarrow$ very ${ }^{*}$ simple: four arithmetic operations

- Interrupts and System Calls

- Process structure, running, terminating, and Process Control Block (PCB)

- Resource, IO devices, and Resource Control Block (RCB)

\footnotetext{
${ }^{1}$ Very, very very
} 
- Process States (even): new, ready, run, blocked, suspend ready/blocked, exit

- Scheduling, Timing, Round-Robin algorithm, Queues

- OS tasks:
○ Process creation (linker $\rightarrow$ "load module")
$\circ$ Process loading (loader $\rightarrow$ memory, DLLs)
- Process context-switch in/out of the CPU
○ Instructing IO devices
○ Handling devices interrupts
- Suspending a Process out of memory
- Reloading suspended process into memory
○ Process termination and cleanup

\section{Simulators (two levels)}

The $1^{\text {st }}$ level simulator is a simple scheduler that manages several processes. The processes may need the CPU or service from one of four different IO devices. The simulator shows how the processes are kept in the READY and four IO queues. The scheduler allocates the CPU time among them using the "Round Robin" algorithm. Processes that need service from IO devices are BLOCKED in the IO queues and are serviced according to the First-Come-First-Serve method.

The $2^{\text {nd }}$ level simulator is an elaborate "scheduler" that shows the details of the OS execution, how it loads a process from the disk to memory, performs context switching, schedules IO devices, communicates with IO devices, handles interrupts, receives blocks, swaps process out of memory (suspend state), swaps them in later and finally "finish" the process. The $2^{\text {nd }}$ simulator shows how the IO controller are working in parallel with the $\mid$ CPU.

\section{Exercise}

We define ten vectors (length $=1000$ ), and fill them with one thousand random numbers. There are 10 processes that should sort the ten vectors using Bubble sort.

Bubble sort consists of repeated rounds on the vector; adjacent elements are compared and swapped if not in order. The algorithm counts the number of swaps in each round, and if the number is zero then it means that the vector is sorted. Using 1000 elements vector with random numbers results on the average with about 120 rounds (although in the worst case scenario it might be 999)

The goal of the project is to write an OS that creates 10 processes (doing bubble sort) and schedules the CPU (execution) among them. Using the Round-Robin algorithm, the OS allocates one sort-round per process to simulate "time-slice".

To simulate using IO we define the following situation: when the process makes more than 500 swaps on the vector it "wants" to "print" it status. In this case the process is taken out of the CPUqueue and placed in the PRINT-queue. When a process is in the first place in the PRINT-queue then it can "print" but it takes 20 time-cycles. 


\section{Simulators}

This section describes the two simulators that run on top of EXCEL.

Simulator $A$

\begin{tabular}{|c|c|c|c|c|c|c|c|c|c|c|c|c|c|c|}
\hline \multirow[b]{2}{*}{ Clock } & $\mathrm{CPU}$ & 101 & 102 & 103 & 104 & P1 & P2 & P3 & P4 & P5 & P6 & P7 & $\mathrm{P} 8$ & P9 \\
\hline & 20 & \multicolumn{3}{|c|}{ CYCLE } & \multicolumn{10}{|c|}{ RESET } \\
\hline Work & 5 & 1 & 1 & 1 & 0 & 2 & 3 & 1 & 2 & 1 & 2 & 1 & 2 & 1 \\
\hline IDLE & 0 & 14 & 15 & 11 & 20 & 17 & 17 & 19 & 17 & 18 & 18 & 18 & 18 & 18 \\
\hline CPU & 20 & & & & & 3 & 3 & 1 & 3 & 2 & 2 & 2 & 2 & 2 \\
\hline 10 & & 6 & 5 & 9 & 0 & 3 & 6 & 0 & 0 & 0 & 6 & 0 & 5 & 0 \\
\hline FINISH & & & & & & 0 & 0 & 0 & 0 & 0 & 0 & 0 & 0 & 0 \\
\hline \multirow[t]{9}{*}{0} & & & & & & \multirow{2}{*}{\multicolumn{9}{|c|}{ Simulator A - by Shimon Cohen }} \\
\hline & \multicolumn{5}{|c|}{ Queues } & & & & & & & & & \\
\hline & P3 1 & P6 2 & P8 3 & P1 4 & & CPU 3 & CPU 2 & CPU 2 & CPU 3 & CPU 4 & CPU 2 & CPU 6 & CPU 2 & CPU 3 \\
\hline & P5 2 & P4 2 & & & & 1037 & 1036 & 1017 & 1012 & 1012 & 1018 & 1029 & 1028 & 1048 \\
\hline & P7 4 & & & & & CPU 2 & CPU 4 & CPU 3 & 1038 & CPU 1 & CPU 1 & CPU 1 & CPU 2 & CPU 2 \\
\hline & P9 1 & & & & & 1027 & 1027 & 1027 & 1027 & 1027 & 1027 & 1017 & 1047 & 1027 \\
\hline & P2 3 & & & & & CPU 1 & CPU 1 & CPU 1 & CPU 1 & CPU 1 & CPU 1 & CPU 1 & CPU 1 & CPU 1 \\
\hline & & & & & & 1039 & 1039 & 1039 & 1039 & 1039 & 1039 & 1039 & 1038 & 1039 \\
\hline & & & & & & CPU 2 & \begin{tabular}{|l|} 
CPU 2 \\
\end{tabular} & CPU 2 & CPU 2 & CPU 2 & CPU 2 & CPU 2 & CPU 2 & CPU 2 \\
\hline
\end{tabular}

Line $1 \rightarrow$ header with names: one CPU, four IO and nine Processes

Line $2 \rightarrow$ The clock (Yellow) and two buttons:

- RESET - to start (or restart) the simulation

- CYCLE - execute one time-cycle

The area below the "Simulator A ..." banner

- $\quad 1^{\text {st }}$ column - Processes in the "READY" queue, each in need of CPU cycles, the first P3 needs 1 Cycle, the second P5 needs 2 more cycles , ...

- Columns 2-5 - show processes waiting for IO service, P6 is serviced by IO1 needs 2 more cycles to finish. Process P4 waits in the queue (needs 2 cycles)..

- Columns 6-16-show the scenarios of 1-9 processes

- $\mathrm{CPU}<\mathrm{n}>-$ needs $<$ n $>$ cycles of CPU

O IO $<$ a $><$ n $>$ - needs IO $<$ a $>$ service for $<$ n $>$ cycles

Line "CPU" $\rightarrow$ number of CPU cycles allocated to each process

Line "IO" $\rightarrow$ number of IO cycles allocated to each process

Line "IDLE" $\rightarrow$ number of "idle" cycles of each process

Line "FINISH" $\rightarrow$ clock-time when process finished its scenario

Line "WORK" $\rightarrow$

- $1^{\text {st }}$ column "CPU” - which process is serviced (Round Robin) 
- $\quad 2-5$ columns - " 1 " busy servicing $1^{\text {st }}$ process in queue, " 0 " - idle

- 6-15 columns - number of item in the scenario (item color is red)

How to use Simulator A

- Setup the processes scenarios - you may leave some of the scenarios empty.

- Click on the "RESET" button

- Click the "CYCLE" button to "execute" one cycle

- Repeat the previous step ("CYCLE" click) until all processes reach the FINISH line.

How to view the screen of Simulator A

- In the beginning all processes need the CPU $\rightarrow$ all in the "CPU" queue. Each process gets one CPU-Cycle (Round Robin)

- When a process finishes using the CPU it needs IO service, the scheduler moves it to the appropriate IO queue.

- In the IO queue $\rightarrow$ only the $1^{\text {st }}$ process is serviced, the other wait in line.

- The $\log$ (below) is produced automatically by the simulator

○ $1^{\text {st }}$ column - clock

○ $2^{\text {nd }}$ column- time needed for the task

- $3^{\text {rd }}$ column - Description 


\begin{tabular}{|l|l|l|}
17 & 7 & $\mathrm{P} 1=>>>$ start IO3 \\
\hline 17 & 4 & $\mathrm{P} 2=\$ \$ \$ \mathrm{~T}=4 \mathrm{~K}=5$ put in $\mathrm{Q}=>\mathrm{CPU}$ \\
\hline 17 & 0 & $\mathrm{P} 2=<<<$ finish IO3 \\
\hline 17 & 2 & $\mathrm{P} 4=\$ \$ \$ \mathrm{~T}=2 \mathrm{~K}=2$ put in $\mathrm{Q} \mathrm{IO} 1$ \\
\hline 17 & 0 & $\mathrm{P} 4=<<<$ finish CPU \\
\hline 16 & 7 & $\mathrm{P} 1=\$ \$ \$ \mathrm{~T}=7 \mathrm{~K}=2$ put in $\mathrm{Q} \mathrm{IO} 3$ \\
\hline 16 & 0 & $\mathrm{P} 1=<<<$ finish $\mathrm{CPU}$ \\
\hline 15 & 8 & $\mathrm{P} 8=>>>$ start IO2 \\
\hline 15 & 8 & $\mathrm{P} 8=\$ \$ \$ \mathrm{~T}=8 \mathrm{~K}=1$ put in $\mathrm{Q} \mathrm{IO} 2$ \\
\hline 15 & 0 & $\mathrm{P} 8=<<<$ finish $\mathrm{CPU}$ \\
\hline 14 & 8 & $\mathrm{P} 6=>>>$ start $\mathrm{IO} 1$ \\
\hline 14 & 8 & $\mathrm{P} 6=\$ \$ \$ \mathrm{~T}=8 \mathrm{~K}=1$ put in $\mathrm{Q} \mathrm{IO} 1$ \\
\hline 14 & 0 & $\mathrm{P} 6=<<<$ finish $\mathrm{CPU}$ \\
\hline 11 & 6 & $\mathrm{P} 2=>>>$ start IO3 \\
\hline 11 & 6 & $\mathrm{P} 2=\$ \$ \$ \mathrm{~T}=6 \mathrm{~K}=1$ put in $\mathrm{Q} \mathrm{IO} 3$ \\
\hline 11 & 0 & $\mathrm{P} 2=<<<$ finish $\mathrm{CPU}$ \\
\hline 0 & 3 & $\mathrm{P} 9=\$ \$ \$ \mathrm{~T}=3 \mathrm{~K}=9$ put in $\mathrm{Q}=>\mathrm{CPU}$ \\
\hline 0 & 2 & $\mathrm{P} 8=\$ \$ \$ \mathrm{~T}=2 \mathrm{~K}=8$ put in $\mathrm{Q}=>\mathrm{CPU}$ \\
\hline 0 & 6 & $\mathrm{P} 7=\$ \$ \$ \mathrm{~T}=6 \mathrm{~K}=7$ put in $\mathrm{Q}=>\mathrm{CPU}$ \\
\hline 0 & 2 & $\mathrm{P} 6=\$ \$ \$ \mathrm{~T}=2 \mathrm{~K}=6$ put in $\mathrm{Q}=>\mathrm{CPU}$ \\
\hline 0 & 4 & $\mathrm{P} 5=\$ \$ \$ \mathrm{~T}=4 \mathrm{~K}=5$ put in $\mathrm{Q}=>\mathrm{CPU}$ \\
\hline 0 & 3 & $\mathrm{P} 4=\$ \$ \$ \mathrm{~T}=3 \mathrm{~K}=4$ put in $\mathrm{Q}=>\mathrm{CPU}$ \\
\hline 0 & 2 & $\mathrm{P} 3=\$ \$ \$ \mathrm{~T}=2 \mathrm{~K}=3$ put in $\mathrm{Q}=>\mathrm{CPU}$ \\
\hline 0 & 2 & $\mathrm{P} 2=\$ \$ \$ \mathrm{~T}=2 \mathrm{~K}=2$ put in $\mathrm{Q}=>\mathrm{CPU}$ \\
\hline 0 & 3 & $\mathrm{P} 1=\$ \$ \$ \mathrm{~T}=3 \mathrm{~K}=1$ put in $\mathrm{Q}=>\mathrm{CPU}$ \\
\hline
\end{tabular}

$\underline{\text { Simulator A - demonstrate? }}$

- How the processes are waiting in the different queues.

- How one CPU is multiplexing its time among the processes using the Round-Robin algorithm.

- How each IO controller serves the 1st process in its queue.

- How processes are moved between the queues by the scheduler.

- NOTE: Operating System tasks (time) are not counted/simulated in Simulator A

- Simulator B is doing the elaborate job of counting and showing the OS tasks. 


\section{Simulator B}

The simulator screen is divided into the following sections:

- PCBs - [top-left] shows the status, service and other parameters of the nine Processes Control Blocks.

- CPU - [top-right] CPU status and the scenario used (see "Data 7")

- The scenario itself is shown below

- RCBs - [bottom-right] four (4) Resource Control Block, each RCB shows the status of an IO controller.

- Script - [bottom-left] shows the scenarios used in the simulation.

Simulator B main Screen

\begin{tabular}{|c|c|c|c|c|c|c|c|c|c|}
\hline \multirow{2}{*}{\begin{tabular}{l|l} 
CYCLE & RESET \\
ID &
\end{tabular}} & \multicolumn{9}{|c|}{ P C B s } \\
\hline & 1 & 2 & 3 & 4 & 5 & 6 & 7 & 8 & 9 \\
\hline Status & Run & S-Block & Block & S-Block & S-Block & S-Ready & S-Block & Block & S-Ready \\
\hline Service & None & Wait IO & Start IO & Wait IO & \begin{tabular}{|l|} 
Wait IO \\
\end{tabular} & None & \begin{tabular}{|l|} 
Wait IO \\
\end{tabular} & Start 10 & None \\
\hline Swap Type & None & None & None & None & None & None & None & None & None \\
\hline Priority & 2 & 2 & 2 & 2 & 2 & 2 & 2 & 2 & 2 \\
\hline Memory Size & 200 & 300 & 400 & 200 & 300 & 400 & 200 & 300 & 400 \\
\hline In Memory & Yes & No & Yes & No & No & No & No & Yes & No \\
\hline Q Number & 395 & 175 & 359 & 154 & 204 & 94 & 233 & 281 & 124 \\
\hline Top Script & 7 & 6 & 6 & 6 & 6 & 5 & 6 & 6 & 5 \\
\hline \multicolumn{10}{|c|}{ Request } \\
\hline Request & CPU & 10 & 10 & 10 & 10 & CPU & 10 & 10 & CPU \\
\hline Request IO & 0 & 2 & 3 & 4 & 1 & 0 & 3 & 4 & 0 \\
\hline Request Parameter & 70 & 4 & 4 & 4 & 4 & 30 & 4 & 4 & 30 \\
\hline Request Left & 69 & 1 & 4 & 2 & 4 & 30 & 2 & 4 & 30 \\
\hline \multicolumn{10}{|c|}{ Time } \\
\hline Start Time & 10 & 30 & 50 & 10 & 30 & 50 & 10 & 30 & 50 \\
\hline End Time & 0 & 0 & 0 & 0 & 0 & 0 & 0 & 0 & 0 \\
\hline CPU Use & 11 & 15 & 30 & 10 & 15 & 0 & 10 & 15 & 0 \\
\hline IO Use & 200 & 197 & 0 & 213 & 30 & 0 & 142 & 0 & 0 \\
\hline KB Wait & 0 & 0 & 0 & 0 & 0 & 0 & 0 & 0 & 0 \\
\hline Cur Start & 395 & 378 & 359 & 342 & 365 & 94 & 393 & 281 & 124 \\
\hline Cur Time & 30 & 0 & 2 & 0 & 0 & 0 & 0 & 2 & 0 \\
\hline KB Time & 0 & 0 & 0 & 0 & 0 & 0 & 0 & 0 & 0 \\
\hline Live Time & & & & & & & & & \\
\hline \multirow{2}{*}{\multicolumn{10}{|c|}{ CPU \% }} \\
\hline & & & & & & & & & \\
\hline \multirow{3}{*}{$\begin{array}{l}\text { Previous } \\
\text { Current } \\
\text { Next }\end{array}$} & 014 & C 15 & C 30 & C 10 & C 15 & 550 & C 10 & C 15 & $S 50$ \\
\hline & C 70 & O24 & O34 & O4 4 & 014 & C 30 & O34 & O4 4 & C 30 \\
\hline & & C 20 & C 90 & C 70 & C 20 & $\mathrm{O} 24$ & C 70 & C 20 & K 200 \\
\hline
\end{tabular}

\begin{tabular}{|l|c|c|c|}
\hline \multicolumn{2}{|c|}{ C P U } & Data & 7 \\
\hline Clock & 395 & 100 & \\
\cline { 1 - 2 } Status & RUN & \multicolumn{1}{|c}{} \\
\cline { 1 - 2 } PCB & 1 & \multicolumn{1}{|c}{} \\
\cline { 1 - 2 } Memory & 1000 & \multicolumn{1}{|c}{} \\
\cline { 1 - 2 } Free & 100 & \\
\hline Run & 106 & $27 \%$ \\
\hline Kernel & 279 & $71 \%$ \\
\hline IDLE & 10 & $3 \%$ \\
\cline { 1 - 2 }
\end{tabular}

Line "ID" $\rightarrow$ PCB numbers

Line "Status" $\rightarrow$ RUN, READY, BLOCKED S-BLOCK (Suspend=Block) ...

Line "Service" $\rightarrow$ Process is waiting for OS service: Wait-IO, Start-IO ...

Line "Swap Type" $\rightarrow$ used when the processes is swap out to a suspend state

Line "Memory Size" $\rightarrow$ How much memory is needed

Line (4 lines) "Request" $\rightarrow$ Parameters for the current service request

Line "Time" $\rightarrow$ Cycles used in various tasks

Line "Cur Start" $\rightarrow$ Clock when current task started

Line "Cur Time" (in red) $\rightarrow$ cycles needed to complete current task

$\underline{\mathrm{RCBs} \text { (four gray squares on the right) }}$

- $\quad \mathrm{RCB}<$ number $>$

- Status: Work, IDLE 
- Per Block: Time needed to access one block on the disk

- PCB: working for PCB

- Total T: total time working

- Wait: time waiting for OS to handle interrupts

- TIME: Time remains to access the current block

\section{Simulator B Scenario}

The scenario instructions are:

- $\mathrm{M}<$ size $>$ - size of process in memory

- $\mathrm{P}<\mathrm{n}>$ - Process priority

- $\mathrm{S}<$ cycles $>-$ when to start the process

- $\mathrm{C}<$ cycles $>$ - CPU cycles needed at this stage

- $\mathrm{O}<\mathrm{n}><$ cycles $>$ - need $<$ cycles $>$ service from IO number $<\mathrm{n}>$

\begin{tabular}{|l|l|l|l|l|l|l|l|l|}
\hline \$ P1 & \$ P2 & $\mathbf{\$ ~ P 3 ~}$ & $\mathbf{\$}$ P4 & $\mathbf{\$}$ P5 & $\mathbf{\$}$ P6 & $\mathbf{\$ ~ P 7}$ & $\mathbf{\$}$ P8 & \$ P9 \\
\hline M 200 & M 300 & M 400 & M 200 & M 300 & M 400 & M 200 & M 300 & M 400 \\
\hline P 2 & P 2 & P 2 & P 2 & P 2 & P 2 & P 2 & P 2 & P 2 \\
\hline S 10 & S 30 & S 50 & S 10 & S 30 & S 50 & S 10 & S 30 & S 50 \\
\hline C 10 & C 15 & C 30 & C 10 & C 15 & C 30 & C 10 & C 15 & C 30 \\
\hline O1 4 & O2 4 & O3 4 & O4 4 & O1 4 & O2 4 & O3 4 & O4 4 & K 200 \\
\hline C 70 & C 20 & C 90 & C 70 & C 20 & C 90 & C 70 & C 20 & C 90 \\
\hline
\end{tabular}

How to use Simulator B

- Setup the scenario in a different sheet (in this example "DATA7")

- Enter the number " 7 " in the blue cell (top-right)

- Click the "RESET" button to initialize the simulator

- Click the "CYCLE" $\rightarrow$ Clock is running (one or more cycles) until the next event.

How to view the screen of Simulator A

- Watch what happens with the processes in the first three PCBs lines: "Status", "Service" and "Swap Type", and the how time of current task "CUR Time" in red is changing.

- Watch the CPU counters on the right

- Watch the RCBs statuses as they work for different PCBs

Simulator B - demonstrate?

- How the OS is using the CPU in order to service the $\mathrm{pCBs}$, there are various services each need the sole attention of the CPU.

- How the RCBs (IO controller) works in parallel to the CPU 


\section{Simulator B parameters}

The tables below show various numbers used to control the simulation. For example: if the OS needs to start an IO operation (line 7 "Start IO") it takes 2 simulator cycles.

\begin{tabular}{|l|l|c|}
\hline \multicolumn{2}{|c|}{ Service } & Time \\
\hline None & None & $\mathbf{0}$ \\
Create & Create Process & $\mathbf{1 0}$ \\
Close & Close Process & $\mathbf{8}$ \\
Start KB & Start KB & $\mathbf{1}$ \\
Wait KB & Suspend if KB > & $\mathbf{7 0}$ \\
INT KB & Handle KB & $\mathbf{1}$ \\
Start IO & Start IO Operation & $\mathbf{2}$ \\
Wait IO & Suspend if IO > & $\mathbf{1}$ \\
INT IO & Handle IO Interrupt & $\mathbf{4}$ \\
Priority & Priority Change & $\mathbf{3}$ \\
Memory & Memory Change & $\mathbf{5}$ \\
\hline
\end{tabular}

\begin{tabular}{|l|l|l|}
\hline \multicolumn{2}{|c|}{ Swap } & \\
\hline None & None & $\mathbf{0}$ \\
Load IN & Load Into Memory & $\mathbf{6}$ \\
L OUT & Load OUT of memory & $\mathbf{9}$ \\
S-IN & Swap Into CPU & $\mathbf{2}$ \\
S-OUT & Swap OUT of CPU & $\mathbf{2}$ \\
\hline
\end{tabular}




\section{Simulator B - Log File}

Detailed Log File:

\begin{tabular}{|r|r|l|}
\hline 395 & 30 & CPU doing P1 Status=Run T=30 \\
\hline 395 & 30 & P1 \$ Status is = Run \\
\hline 393 & 2 & CPU doing P1 Status=Ready Swap=S-IN T=2 \\
\hline 393 & 2 & P1 * Swap Request is = S-IN \\
\hline 393 & 70 & IO3 handles a Block for P7 \\
\hline 393 & 0 & P7 = Service IO Next R3 Blocks=2 of 4 \\
\hline 389 & 4 & CPU doing P7 Status=S-Block Service=INT IO T=4 \\
\hline 389 & 0 & P1 \$ Status is = Ready \\
\hline 387 & 2 & CPU doing P1 Status=Run Swap=S-OUT T=2 \\
\hline 387 & 2 & P1 * Swap Request is = S-OUT \\
\hline 387 & 4 & P7 = Service Request is = INT IO \\
\hline 387 & 0 & IO3 Interrupt for P7 !!! \\
\hline 386 & 30 & CPU doing P1 Status=Run T=30 \\
\hline 386 & 30 & P1 \$ Status is = Run \\
\hline 384 & 2 & CPU doing P1 Status=Ready Swap=S-IN T=2 \\
\hline 384 & 2 & P1 * Swap Request is = S-IN \\
\hline 384 & 0 & P1 \$ Status is = Ready \\
\hline 378 & 6 & CPU doing P1 Status=S-Ready Swap=Load IN T=6 \\
\hline 378 & 6 & P1 * Swap Request is = Load IN \\
\hline 378 & 60 & IO2 handles a Block for P2 \\
\hline 378 & 0 & P2 = Service IO Next R2 Blocks=1 of 4 \\
\hline 374 & 4 & CPU doing P2 Status=S-Block Service=INT IO T=4 \\
\hline 373 & 4 & P2 = Service Request is = INT IO \\
\hline 373 & 0 & IO2 Interrupt for P2 !!! \\
\hline 365 & 9 & CPU doing P5 Status=Block Service=Wait IO Swap=L OUT T=9 \\
\hline 365 & 9 & P5 * Swap Request is = L OUT \\
\hline & & \\
\hline 389 & &
\end{tabular}

\section{Conclusion}

Undergraduate students find it difficult to comprehend the concepts of the operating systems. There are many new ideas, buzz-words, multiple tasks and "strange" algorithms. Using the proposed method (see below) shows that the students experience and reaction to the course was changed from "dull" / "too complicated" to "interesting" and "challenging". The use of the simulators coupled with the exercise resulted in much better understanding of the scheduler (as tested in the mid-term) 
1. Introduction to OS, Interrupts, Processes States

2. Show the simulator $\mathrm{A}$

3. Explain System Calls, Timing and Scheduling

4. Show the simulator B

5. Exercise

\section{References}

Dietel, H. M. (1992). Operating systems (2nd ed.). Reading, MA: Addison-Wesley.

Finkel, R.A. (1988). An operating systems vade mecum", 2nd ed. Prentice-Hall, Englewood Cliffs, NJ,.

Goscinski, A. (1991). Distributed operating systems: The logical design.

Hartley, S. J. (1994). Operating systems programming.

Krakowiak, S. (1988). Principles of operating systems. MIT Press.

Lane, M. G., \& Mooney, J. D. (1988). A practical approach to operating systems. Boyd and Fraser.

Milenkovic, M. (1992). Operating systems: Concept and design (2nd ed.). McGraw-Hill.

Silberschatz, A., \& Galvin, P.B. (1994). Operating system concepts (4th ed.). Reading, MA: AddisonWesley.

Tanenbaum, A. S. (1987). Operating systems: Design and implementation.

Tanenbaum, A. S. (1992). Modern operating systems. Englewood Cliffs, NJ: Prentice-Hall.

Tel, G. (2000). Introduction to distributed algorithms.

\section{Biography}

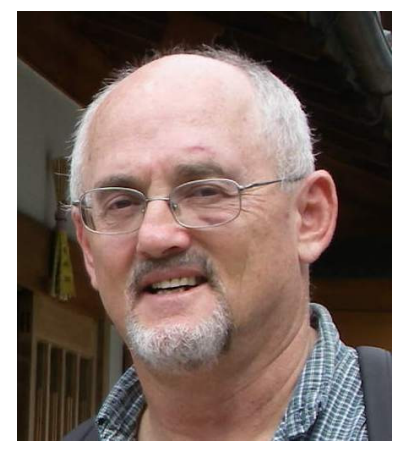

Shimon Cohen has been working in the Israeli Hi-Tech Industry for the last 30 years, including projects in areas of: Databases, Parallel computing, Artificial Intelligence, E-Learning, Geo-Graphical information, Cellular Communication. Teaching Operating Systems, Object Oriented Programming, Artificial Intelligence, and other Computer Science courses. 